
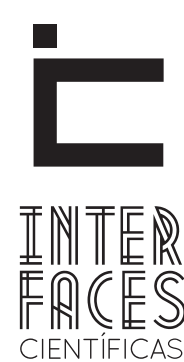

SAÚDE E AMBIENTE

\title{
ANÁLISE DA PREMATURIDADE NOS EXTREMOS DA IDADE REPRODUTIVA
}

\author{
Larissa Beatriz Bomfim dos Santos ${ }^{1}$ \\ Alba Maria Bomfim de França ${ }^{3}$ \\ Fernanda Demutti Pimpão ${ }^{5}$
}

Mayra Paula Santos Castela ${ }^{2}$

Ana Paula Rebelo Aquino Rodrigues ${ }^{4}$

\section{RESUMO}

A prematuridade é decorrente de circunstâncias diversas e, por vezes, imprevisíveis associadas a fatores epidemiológicos e obstétricos, acarretando assim um alto custo social e financeiro. A prematuridade é definida como aquela em que a gravidez se interrompe antes da $37^{\mathrm{a}}$ semana de idade gestacional, mais precisamente entre a $22^{\mathrm{a}}$ e a $36^{\mathrm{a}}$ semana e 6 dias de gestação. Neste sentido, o estudo tem como objetivo analisar os fatores associados à prematuridade nos extremos da idade reprodutiva. Trata-se de um estudo transversal, descritivo, retrospectivo, de campo com abordagem quantitativa. A pesquisa foi realizada na Maternidade Escola Santa Mônica (MESM) composta de 139 prontuários de mulheres nos extremos da idade reprodutiva que tiveram partos prematuros. Os dados foram coletados entre os meses de fevereiro a abril de 2014 por intermédio de questionários preenchidos pelas pesquisadoras, foram incluídas na pesquisa prontuários de mulheres nos extremos da idade reprodutiva que tiveram partos prematuros, na MESM no ano de 2012. Observou-se que das 139 mulheres que compõem a amostra, 118 eram adolescentes, dessas $86 \%$ primíparas, enquanto as mulheres acima dos 35 anos (21 pacientes), 20\% das mesmas encontravam-se na quinta gestação (multíparas). Quanto à idade gestacional até o momento do parto, as maiores taxas de ambos os extremos se mantiveram entre 34 e 36 semanas, sendo $60 \%$ das adolescentes e $38 \%$ das gestações tardias. Constatou-se que as variáveis relacionadas à idade que implicaram no trabalho de parto prematuro foram aborto prévio, primiparidade, sofrimento fetal, infecções do trato urinário e cobertura pré-natal inadequada.

\section{DESCRITORES}

Enfermagem, Parto Prematuro, Idade Materna. 


\section{ABSTRACT}

Prematurity is due to several circumstances and sometimes unpredictable associated with epidemiological and obstetric factors, thus causing a high social and financial cost. Prematurity is defined as one in which the pregnancy is interrupted before 37 weeks of gestational age, more precisely between 22 and 36 weeks and 6 days of gestation. In this sense, the study aims to analyze the factors associated with prematurity in the reproductive age extremes. This is a cross-sectional, descriptive, retrospective study field with quantitative approach. The survey was conducted in the Maternity Hospital Santa Monica (EVEN) composed of 139 medical records of women in the reproductive age who had extreme premature births. Data were collected between February and April 2014 through questionnaires filled out by the researchers, were included in the women's medical records in search of reproductive age who had extreme premature births in EVEN in 2012. It was observed that of 139 women in the sample, 118 were teenagers, $86 \%$ of these gilts, while women over 35 years (21 patients), 20\% of them were in the fifth pregnancy (multiparous). As for gestational age until the time of delivery, the highest rates of both ends were between 34 and 36 weeks, $60 \%$ of girls and $38 \%$ of late pregnancies. It was found that the variables related to age involving in preterm labor were previous abortion, primiparity, fetal distress, urinary tract infections and inadequate prenatal coverage.

\section{KEYWORDS}

\section{Nursing. Premature Birth. Maternal Age.}

\section{RESUMEN}

La prematuridad es debido a las varias circunstancias, y muchas veces, imprevisibles asociada entre otros factores epidemiológicos y obstétricos, en todas las clases sociales, lo que causa un costo social y financiero muy altos. La prematuridad se define como aquella en el que el embarazo se interrumpe antes de las 37 semanas de edad gestacional, más precisamente entre 22 y 36 semanas y 6 días de gestación. Tratase de un estudio transversal, descriptivo, retrospectivo donde se analiza los factores asociados a la prematuridad en los extremos de la edad reproductiva. La encuesta se realizó en el Hospital Maternidad Santa Mónica (HSHAV) compuesto de 139 historias clínicas de las mujeres en edad reproductiva que tuvieron partos prematuros extremos. Los datos fueron colectados entre febrero y abril 2014 a través de cuestionarios cumplimentados por los investigadores, y fueron incluidos en la historia clínica de la mujer, bus- cando en los extremos de la edad reproductiva que han tenido partos prematuros, en HSHAV en 2012. Se observó que 139 mujeres de la muestra, 118 eran adolescentes, o sea, $86 \%$ eran primíparas, mientras que las mujeres mayores de 35 años (21 pacientes), 20\% de ellos estaban en el quinto embarazo (multíparas). En cuanto a la edad gestacional hasta el momento del parto, las tasas más altas de ambos extremos tenían entre 34 y 36 semanas, el $60 \%$ muy son jóvenes, y $38 \%$ embarazos tardíos. Se encontró que variables relacionadas con la edad, que dio lugar a un parto prematuro eran aborto anterior, sufrimiento fetal, infecciones del tracto urinario y la cobertura pre natal inadecuada.

\section{DESCRIPTORES}

Ancianos, Parto Prematuro, La Edad Materna. 


\section{INTRODUÇÃO}

A gravidez é um evento biologicamente natural, porém, especial na vida da mulher e, como tal, desenvolve-se em um contexto social e cultural que influencia e determina a sua evolução e a sua ocorrência. Para tanto, deve ser vista, pelas gestantes e profissionais de saúde, como uma experiência de vida saudável, mas que por vezes pode implicar riscos tanto para a mãe quanto para o feto (BRASIL, 2010).

A prematuridade é definida como aquela em que a gravidez se interrompe antes da $37^{\mathrm{a}}$ semana de idade gestacional, mais precisamente entre a $22^{\mathrm{a}}$ e a $36^{\mathrm{a}}$ semanas e seis dias. Sendo ela uma das principais causas de morbidade e mortalidade a curto e longo prazo, merecendo uma maior atenção a fim de preveni-la (NEME, 2005).

Estudos afirmam que a incidência mundial de nascimento pré-termo aquilatada para o ano de 2005 foi de $9,6 \%$, e as maiores taxas foram observadas nos Estados Unidos da América (10,6\%) e em países da África (11,9\%), e as menores, em países da Europa (6,2\%). A prematuridade afeta desproporcionalmente os países em desenvolvimento, principalmente os da Ásia e África. No Brasil, a tendência de aumento dos pré-termos acompanha o panorama mundial. Estudo de base populacional, nacionais com dados primários, mostrou aumento de $4 \%$ para $12 \%$ da prevalência, em diferentes contextos, entre 1980 e 2000 (ASSUNÇÃO ET AL., 2012).

O parto pré-termo ou prematuro resulta de um conjunto de fatores inter-relacionados e traz, anualmente, aos países um alto custo social e econômico, uma vez que impacta significantemente na mortalidade infantil e na qualidade de vida dos que sobrevivem com sequelas (ASSUNÇÃO ET AL., 2012).

No Brasil, em 2000, os adolescentes representavam um quinto da população total do país, segundo o
Instituto Brasileiro de Geografia e Estatística (IBGE) e o percentual de nascidos vivos de mulheres com menos de 20 anos em 2005 representou $21,6 \%$ do total, taxa variável entre os estados, sendo as menores no Distrito Federal (15,8\%) e em São Paulo (16,9\%), e as maiores no Maranhão (30,2\%) e no Pará (29,9\%). Apesar dos esforços governamentais, a taxa deste agravo na adolescência tem se mantido entre a população de 15 a 19 anos e vem aumentado na população de 10 a 14 anos (SANTOS ET AL., 2009).

\begin{abstract}
Entre as mulheres de 15 a 19 anos, a chance de ocorrência de morte por problemas decorrentes da gravidez ou do parto é duas vezes maior do que entre as maiores de 20 anos, e entre as menores de 15, essa ocorrência é ainda cinco vezes maior, sendo uma das principais causas de morte da faixa etária. (CARNIEL ET AL., 2006, p. 420).
\end{abstract}

Como agravante de uma gravidez na adolescência, configura-se a não realização ou a má qualidade do pré-natal ou um pré-natal de má qualidade, visto que algumas complicações são muito mais frequentes nas grávidas adolescentes que não tiveram assistência pré-natal, perdendo assim a oportunidade de detecção e intervenção precoce a fim de amenizar os fatores de risco determinantes. 0 aumento na taxa de incidência de gestações precoces confirma o aumento da escala de idade fértil, considerada hoje em dia entre 10 e 49 anos, de acordo com os dados da Pesquisa Nacional de Demografia e Saúde (PNDS, 2006).

Em países desenvolvidos, esse aumento no número de adolescentes grávidas, desperta bastante interesse entre profissionais da saúde e pesquisadores, pois é comum ocorrer entre adolescentes a interrupção da escolaridade e da formação profissional em decorrência da gravidez. Tal situação, acarretando dificuldades de inserção da adolescente no mercado de trabalho, perpetuando à tendência a pobreza, trazendo riscos sociais para a mãe e para seus dependentes. 
No outro extremo reprodutivo, têm-se as primíparas idosas, nomenclatura designada pelo conselho da Federação Nacional de Ginecologia e Obstetrícia definiu em 1958, cuja definição consiste em gestantes como primíparas idosas as pacientes com 35 anos ou mais, essas são consideradas atualmente gestações tardias. Entretanto, muitos ainda subdividem o grupo entre mulheres até 40 anos e com mais de 40 anos, por ser evidente o aumento do risco materno e perinatal ultrapassada a quarta década de vida da mulher (SANTOS ET AL., 2009).

Dados de 2006 da Empresa de Processamento de Dados da Previdência Social (DATASUS) mostram um aumento de 7,9 para 9,6\% de gestações nesse período da vida. Em países desenvolvidos, a gestação em mulheres acima de 35 anos aumentou substancialmente, como nos Estados Unidos em 2005, em que $14,4 \%$ das gestações foram de mulheres acima de 35 anos. Na última década, o número de gestantes primíparas com 30 ou mais anos dobrou e os casos de grávidas com mais de 40 anos aumentaram em $80 \%$ (DATASUS, 2006).

As mulheres que engravidam mais tardiamente, em grande parte dos casos, apresentam ótimo estado de saúde, gestações planejadas e desejadas, muitas vezes de reprodução assistida, não raro pela redução de fertilidade que a idade representa. Além desse grupo, que seria o de menor risco e melhores condições socioeconômicas, entre as gestantes tardias estão, também, aquelas que foram mães mais cedo e agora são multíparas, com ou sem comorbidades associadas, e que se tornam um contingente vulnerável a merecer atenção especializada (SANTOS ET AL., 2009)

As mulheres com gestação tardia apresentam risco similar às adolescentes em alguns aspectos, e mais elevado em outras situações como o abortamento espontâneo, gravidez ectópica, anormalidades cromossômicas e malformações congênitas. Alguns trabalhos demonstram que a gravidez na idade avançada, também, se associa a complicações fetais, como anomalias cromossômicas, sofrimento fetal, internação em Unidades de Terapia Intensiva (UTI), e óbito neonatal, baixo peso ao nascer e restrição do crescimento fetal, além de taxas de inexplicável perda fetal tardia, mais pronunciada entre mulheres com 35 anos ou mais (SANTOS ET AL., 2009).

Então, é relevante responder à pergunta de pesquisa: quais são os fatores de risco associados à prematuridade nos extremos da idade reprodutiva? Visto que a incidência de prematuridade nas gestações situadas nos extremos da idade reprodutiva representa um significativo problema de saúde pública. Acredita-se que os fatores de risco associados à prematuridade, apesar de serem os mesmos afetam diferentemente os extremos de idade reprodutiva.

Assim, este estudo preocupou-se em analisar os fatores associados à prematuridade nos extremos da idade reprodutiva, identificando o perfil sociodemográfico das adolescentes e mulheres acima de 35 anos que tiveram parto prematuro, os fatores de risco (sociais, clínicos e obstétricos) presentes nas adolescentes e mulheres acima de 35 anos que tiveram parto prematuro, assim como a incidência distribuição dos partos prematuros e a classificação da prematuridade em adolescentes e em mulheres acima de 35.

\section{MÉTODOS}

Trata-se de uma pesquisa de campo descritiva, retrospectiva com abordagem quantitativa. 0 estudo foi realizado na instituição pública de referência para alto risco Maternidade Escola Santa Mônica (MESM), localizada no município de Maceió-AL, no $1^{\circ}$ distrito.

Trata-se de um estudo com características censitárias, para tanto, foram consultados todos os prontuários de mulheres nos extremos da idade reprodutiva que tiveram partos prematuros, de janeiro a dezembro de 2012, na MESM, totalizando 139 prontuários. Foram incluídos na pesquisa prontuários de mulheres 10 
a 19 anos ou acima dos 35 anos, os extremos da idade reprodutiva que tiveram partos prematuros, de 22 a 36 semanas e seis dias, na Maternidade Escola Santa Mônica no ano de 2012. Foram excluídos da pesquisa prontuários com o preenchimento deficiente ou que estavam sob a responsabilidade de outros setores.

Para a pesquisa foi utilizado um instrumento para identificar em duas partes a população do estudo, a primeira, abordando as variáveis primarias: idade materna e idade gestacional do parto e variáveis secundárias: estado civil, naturalidade e residência atual, intercorrências clínicas e obstétricas nas gestações prévias e na gestação atual, histórico obstétrico $\left(n^{\circ}\right.$ de gestações, parto e aborto) e número de consultas pré-natal.

A coleta de dados foi realizada no período de fevereiro a abril de 2014. Inicialmente foram consultados os registros de admissão dos pacientes e identificados os prontuários de mulheres que internaram por trabalho de parto prematuro, sendo coletados: nome, número do prontuário e idade da paciente. Após essa etapa os prontuários foram classificados de acordo com os extremos de idade reprodutiva e posteriormente consultados para verificar os critérios de inclusão em relação à ocorrência de prematuridade de acordo com a idade gestacional.
Em posse dessas informações iniciou-se a coleta de dados no prontuário, de acordo com o instrumento elaborado para fins desta pesquisa. A consulta aos prontuários foi realizada no arquivo da MESM, sendo consultados apenas 25 prontuários por dia, com dados registrados de forma sequencial, iniciando do numeral um, também, no sentido de garantir que as informações fossem precisas e verídicas.

A tabulação e análise dos dados foram realizadas por meio de uma análise descritiva simples em porcentagem com o auxílio do programa Microsoft Office Excel ${ }^{\circledR} 2010$. Os resultados são apresentados por meio de tabelas e discutidos à luz de autores da área e experiência das pesquisadoras, os dados serão apresentados por meio de Figuras e Gráficos. 0 sigilo e o anonimato das pacientes foram respeitados, de forma que as informações coletadas nos prontuários foram utilizadas somente para os objetivos do estudo e sem identificar a identidade das mulheres, sendo as pacientes identificadas pela "P” de prontuário seguido do $n^{0}$ de ordem da coleta.

No sentido de garantir que os princípios éticos e bioéticos fossem respeitados o projeto de pesquisa foi encaminhado ao Comitê de Ética em Pesquisa da Universidade Estadual de Ciências da Saúde de Alagoas (UNCISAL), e deu-se início à coleta de dados somente após sua aprovação (protocolo: 1787 de 14/10/2011). 


\section{RESULTADOS}

Tabela 1 - Histórico Gestacional de adolescentes e mulheres > de 35 anos, que tiveram parto prematuro na MESM no ano de 2012, Maceió - AL, 2014

\begin{tabular}{|c|c|c|c|c|c|c|}
\hline \multirow{2}{*}{ Prematuridade } & \multirow{2}{*}{ Categorias } & \multicolumn{2}{|c|}{ Adolescentes } & \multicolumn{2}{|c|}{$>35$ anos } & \multirow{2}{*}{$P$} \\
\hline & & $\mathrm{n}$ & $\%$ & $\mathrm{~N}$ & $\%$ & \\
\hline \multirow{2}{*}{ Paridade } & Primíparas & 86 & 72,9 & 4 & 19,0 & \multirow{2}{*}{$<0,001$} \\
\hline & Multíparas & 32 & 27,1 & 17 & 81,0 & \\
\hline \multirow{2}{*}{ Histórico de doenças } & Sim & 2 & 1,7 & 1 & 6,7 & \multirow{2}{*}{0,304} \\
\hline & Não & 116 & 98,3 & 14 & 93,3 & \\
\hline \multirow{2}{*}{$\begin{array}{l}\text { Intercorrências em gesta- } \\
\text { ções anteriores }\end{array}$} & Sim & 16 & 13,6 & 8 & 38,1 & \multirow{2}{*}{0,011} \\
\hline & Não & 102 & 86,4 & 13 & 61,9 & \\
\hline \multirow{2}{*}{$\begin{array}{l}\text { Intercorrências na gesta- } \\
\text { ção atual }\end{array}$} & Sim & 51 & 43,2 & 14 & 66,7 & \multirow{2}{*}{0,04} \\
\hline & Não & 67 & 56,8 & 7 & 33,3 & \\
\hline \multirow{3}{*}{$\begin{array}{l}\text { Número de consultas pré- } \\
\text {-natal }\end{array}$} & 1 a 3 & 49 & 41,5 & 6 & 28,6 & \multirow{3}{*}{0,163} \\
\hline & 4 a 6 & 47 & 39,8 & 13 & 61,9 & \\
\hline & Acima de 7 & 22 & 18,6 & 2 & 9,5 & \\
\hline
\end{tabular}

Fonte: Dados da pesquisa, 2014.

A taxa de paridade entre as gestantes foram $72,9 \%$ entre as adolescentes, que eram primíparas, $81 \%$ mulheres acima dos 35 anos eram multíparas $(p<0,001)$. 0 menor número de consultas pré-natal ocorreu entre as adolescentes (41,5\%), a maioria das mulheres acima dos 35 anos realizaram de quatro a seis consultas $(61,9 \%)(p<0,163)$. 
Tabela 2 - Classificação dos partos prematuros em adolescentes e mulheres > de 35 anos, na Maternidade Escola Santa Mônica no ano de 2012, Maceió - AL, 2014

\begin{tabular}{llllll}
\hline \multirow{2}{*}{ Prematuridade } & \multicolumn{2}{l}{ Adolescentes } & \multicolumn{2}{c}{$>$ 35 anos } & P \\
& $\mathrm{n}$ & $\%$ & $\mathrm{n}$ & $\%$ & \\
\hline Extrema < 28 semanas & 8 & 6,80 & 4 & 19,00 & \\
Grave 28 - 30 semanas & 13 & 11,00 & 8 & 38,10 & \\
Moderada 31 - 33 semanas & 26 & 22,00 & 1 & 4,80 & \\
Quase termo 34 - 36 semanas & 71 & 60,20 & 8 & 38,10 & \\
\hline
\end{tabular}

Fonte: Dados da pesquisa, 2014.

Classificação de prematuridade (REZENDE, 2011).

A Tabela 2 apresenta a idade gestacional até 0 momento do parto, as maiores taxas de ambos os extremos se mantiveram entre 34 e 36 semanas, sendo
$60,20 \%$ das adolescentes e $38,10 \%$ das gestações tardias. Pode-se observar, também, que $38,10 \%$ das mulheres acima de 35 anos tiveram prematuridade grave $(p<0,001)$.

Figura 1 - Relação entre as doenças prevalentes na gestação atual entre as adolescentes e mulheres >35 anos na Maternidade Escola Santa Mônica- MESM no ano de 2012, Maceió-AL, 2014

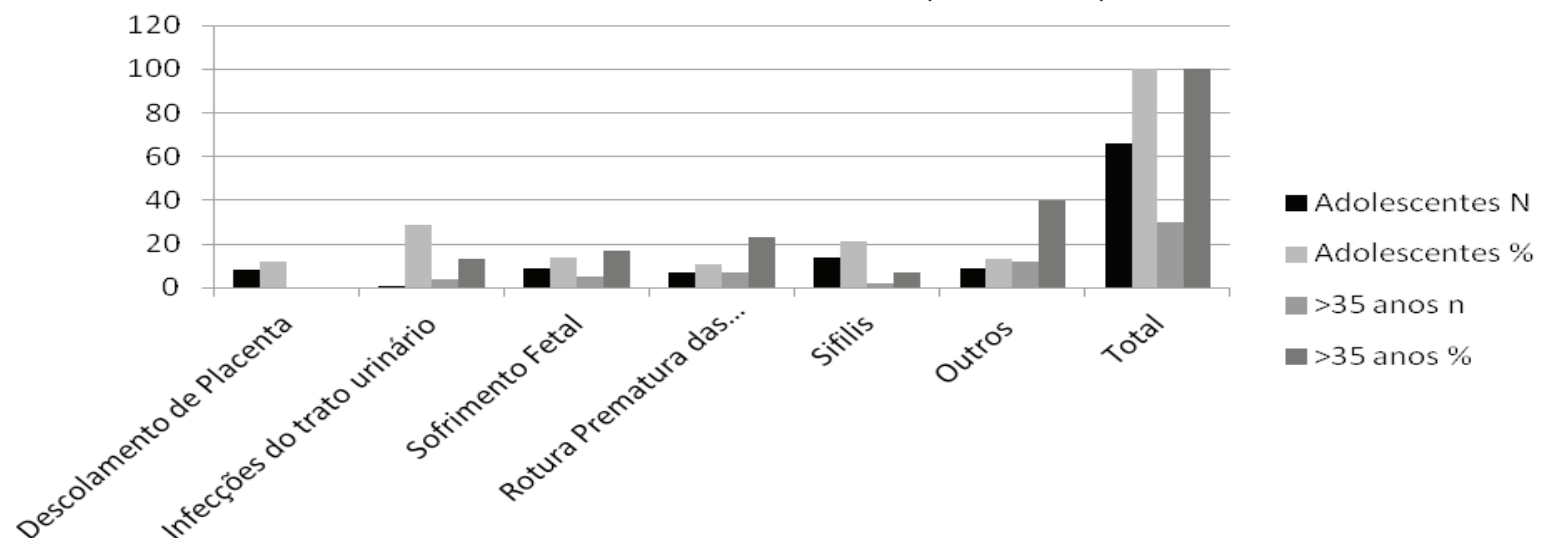

Fonte: Dados da pesquisa, 2014. 
Podem-se analisar os problemas na atual gestação onde os dados mostram que $29 \%$ das adolescentes apresentaram infecções de trato urinário, já nas mulheres acima dos 35 anos a maior frequência foi de rotura prematura de membrana com 23\%. $14 \%$ dos conceptos das adolescentes tiveram sofrimento fetal e $17 \%$ dos conceptos das mulheres acima dos 35 anos. 12\% das adolescen- tes apresentaram descolamento de placenta. Em relação à sífilis 7\% das mulheres acima dos 35 anos tiveram e $21 \%$ das adolescentes. Os problemas gestacionais que não tiveram significância na pergunta foram agrupados e estes são: Varicela, coriomniote, sepse, apendicite, placenta prévia, eclampsia, hipertensão gestacional e diabetes gestacional.

Figura 2 - Taxa de aborto e prematuridade em adolescentes e mulheres > de 35 anos na Maternidade Escola Santa Mônica no ano de 2012, Maceió - AL, 2014

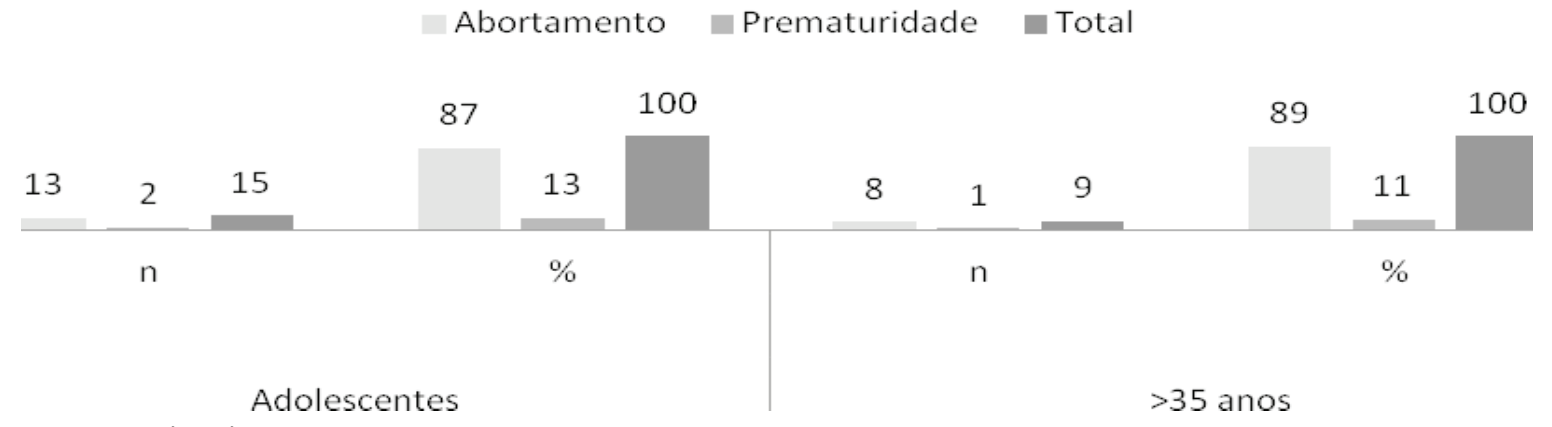

Fonte: Dados da pesquisa, 2014.

Podem-se observar os problemas na gestação anterior, onde $87 \%$ das adolescentes apresentaram aborto e $13 \%$ tiveram parto pré-termo, enquanto que no extremo superior de idade reprodutiva $89 \%$ tiveram aborto e $11 \%$ bebês pre- maturos. As outras opções, (recém nascidos (RN) pequeno para a idade gestacional (PIG), hipertensão induzida pela gravidez (HIG), anemia e outros), não obtiveram resultados significativos para a pesquisa. 
Tabela 5 - Caracterização sociodemográfica das pacientes que tiveram parto prematuro nos extremos da idade reprodutiva no ano de 2012 em uma maternidade de alto risco em Maceió - AL, 2014

\begin{tabular}{|c|c|c|c|c|c|}
\hline Variavél & Categoria & Frequência & Porcentual & $\begin{array}{l}\text { Porcentagem } \\
\text { válida }\end{array}$ & $\begin{array}{l}\text { Porcentagem } \\
\text { acumulativa }\end{array}$ \\
\hline \multirow{5}{*}{ Idade Materna } & $10-15$ & 39 & 28,1 & 28,1 & 28,1 \\
\hline & $16-19$ & 79 & 56,8 & 56,8 & 84,9 \\
\hline & $35-39$ & 18 & 12,9 & 12,9 & 97,8 \\
\hline & $>40$ & 3 & 2,2 & 2,2 & 100 \\
\hline & Total & 139 & 100 & 100 & \\
\hline \multirow{4}{*}{ Estado civil } & Solteira & 63 & 45,3 & 45,3 & 45,3 \\
\hline & União estável & 53 & 38,1 & 38,1 & 83,5 \\
\hline & Casada & 23 & 16,5 & 16,5 & 100 \\
\hline & Total & 139 & 100 & 100 & \\
\hline \multirow{5}{*}{ Naturalidade } & Alagoana & 135 & 97,1 & 97,1 & 97,1 \\
\hline & Paraíba & 1 & 0,7 & 0,7 & 97,8 \\
\hline & Pernambuco & 2 & 1,4 & 1,4 & 99,3 \\
\hline & São Paulo & 1 & 0,7 & 0,7 & 100 \\
\hline & Total & 139 & 100 & 100 & \\
\hline \multirow{5}{*}{ Procedência } & Agreste & 2 & 1,4 & 1,4 & 74,1 \\
\hline & Sertão & 6 & 4,2 & 4,2 & 328,8 \\
\hline & Leste & 41 & 29,2 & 29,2 & 1181,9 \\
\hline & Maceió & 78 & 56,1 & 56,1 & 64 \\
\hline & Total & 127 & 99,9 & 99,9 & \\
\hline
\end{tabular}

Fonte: Dados da pesquisa, 2014. 
Conforme a Tabela, com relação à idade materna a maior frequência foi das adolescentes na faixa etária entre 16 a 19 anos, totalizando 79 (56,8\%), entre as mulheres acima dos 35 anos foram 18 (12,9\%). Quando observado o estado civil a maioria era solteira totalizando $63(45,3 \%)$ e em segundo lugar as que estavam em união estável 53 (38,1\%). 135 das pesquisadas eram natural de alagoas $(97,1 \%)$, destas mais da metade $56,1 \%$ eram residentes no município de Maceió e 41 delas $(28,2 \%)$ eram do leste alagoano.

\section{DISCUSSÃO}

A Maternidade Escola Santa Mônica (MESM) é referência no estado de Alagoas para gestação de alto risco, sendo responsável por grande parte dos atendimentos a gestação de alto risco em todo o estado, portanto, é apta a determinar uma importante e representativa amostra populacional. Segundo as literaturas pesquisadas, as mulheres nas faixas etárias extremas apresentam maior chance de complicações gestacionais e comprometimentos da evolução gestacional e resultado neonatal.

Na pesquisa em questão observou-se que as maiores taxas de nascimento pré-termo foram daquelas em que a idade gestacional situava-se entre $34^{\mathrm{a}}$ e $36^{\mathrm{a}}$ semana de gestação (prematuros quase termo) sendo $60 \%$ das adolescentes e $38 \%$ nas mulheres acima de 35 anos, corroborando com resultados encontrados em outros estudos, onde a grande maioria das pesquisadas ( $80 \%)$ apresentaram partos prematuros com idade gestacional entre $32^{\mathrm{a}}$ e $36^{\mathrm{a}}$ semanas, confirmando que a maioria dos nascimentos prematuros são aqueles quase termos (SILVA ET AL., 2009). Das mulheres que contribuíram, fazendo parte da população da pesquisa, 118 eram adolescentes, dessas $73 \%$ primíparas, já 40\% das mulheres acima dos 35 anos eram multíparas.

Em relação ao abortamento, $47 \%$ das adolescentes apresentaram aborto anterior, contra $46 \%$ das mulheres acima dos 35 anos. No tocante a primiparidade, estudos apontam que a paridade em conjunto com fatores sociodemográficos, tais como idade e baixa escolaridade, pode aumentar o risco de um nascimento prematuro (ALMEIDA ET AL., 2012).

A maior frequência de idades encontrada entre as adolescentes foi entre 16 e 19 anos (56,8\%), nas gestantes tardias a maior frequência foi entre 35 e 39 anos (12,9\%). Um estudo realizado em Porto Alegre, no ano de 2009, mostrou quedas: 331 gestantes que constituíram a população em estudo, 269 (81,3\%) eram adolescentes e $62(18,7 \%)$ gestantes tardias, a idade das adolescentes variou de 13 a 19 anos, enquanto as gestantes tardias possuíam idades entre 35 a 46 anos, concordando com os achados desta pesquisa.

O estado civil da gestante é de fundamental importância, já que a gravidez torna a mulher vulnerável emocional e financeiramente quando ela não tem um companheiro para dividir as responsabilidades advindas com a chegada do bebê. No estudo foi evidenciado que a maioria das pesquisadas eram solteiras, totalizando 45,3\%, contrariando o estudo realizado na Maternidade Publica do Maranhão onde, 50,38\% das gestantes eram casadas enquanto $38,35 \%$ solteiras. As gestações de adolescentes e mulheres acima dos 40 anos propiciaram um maior risco de retardo do crescimento intra-uterino, sofrimento fetal, morte intra-uterina e outras intercorrências (STOLL; KLIEGMAN, 2002).

Notou-se com o estudo que entre os fatores de risco relacionados a problemas na gestação atual houve uma predominância de infecção do trato urinário (29\%) nas adolescentes, concordando com o estudo de Seidler e outros autores (2012) onde a infecção urinária ocorreu em $25 \%$ das pesquisadas. As infecções maternas, incluindo a urinária, que tenham manifestações sistêmicas podem ter como consequência o parto pré-termo, feto natimorto ou aborto. 0 feto muitas vezes pode ser infectado, independente do grau da infecção materna (STOLL; KLIEGMAN, 2002 citado por SEIDLER 2012). 
A rotura prematura de membrana ocorreu principalmente nas mulheres acima dos 35 anos (23\%). Alguns pesquisadores encontram uma proporção maior em relação à rotura prematura de membrana e prematuridade, onde trabalho de parto prematuro (TPP) foi diagnosticado em 103 mulheres (50,7\%), a ruptura prematura pré-termo de membranas (RPM) em 72 (35,5\%) e a associação de ambos em 28 (13,8\%) mulheres (NOMURA ET AL., 2009).

Entre os conceptos das adolescentes 14\% apresentaram sofrimento fetal e $17 \%$ entre os das mutheres acima dos 35 anos. Um estudo realizado em São Paulo evidenciou que $49,5 \%$ das pesquisadas apresentaram parto prematuro eletivo e sofrimento fetal. Em relação à sífilis $7 \%$ das mulheres acima dos 35 anos tiveram e $21 \%$ das adolescentes A sífilis pode levar ao trabalho de parto e nascimento prematuro (AGUIAR, CARDOSO; LUCIO, 2007).

Os problemas gestacionais que não mostraram significância na pesquisa foram agrupados e estes são: Varicela, coriomniote, sepse, apendicite, placenta prévia, eclampsia, hipertensão gestacional e diabetes gestacional, concordando com o estudo realizado no Hospital Geral de Caxias do Sul que está se tornando um centro de referência para gestantes de alto risco e mantém um Ambulatório de Atendimento para as mesmas, por isso alguns resultados obtidos na pesquisa podem ter sido influenciados, associando a prematuridade às síndromes hipertensiva $(n=20 ; 17,1 \%)$, diabética $(n=5 ; 4,3 \%)$, ainda que os percentuais observados estejam majorados em ambos os grupos de estudo (MADI ET AL., 2012).

A literatura destaca a importância do pré-natal como sendo um dos principais determinantes da adequada evolução gestacional, permitindo identificar situações de risco que podem acarretar o trabalho de parto prematuro precocemente. Consequência de uma assistência pré-natal inadequada entre as adolescentes mostram um maior impacto negativo, visto que a gravidez nesta faixa etária acomete com maior afinco as jovens menos favorecidas e por vezes sem apoio social, familiar e do companheiro (SANTOS ET AL., 2014).

O número de consultas pré-natal de maior frequência entre as adolescentes foi de uma a três consultas em um total de $44 \%$ delas. As mulheres acima dos 35 anos 20\% realizaram de quatro a seis consultas. Os achados de menor presença das adolescentes a consulta pré-natal concorda com outros estudos onde as adolescentes apresentam o menor número de consultas pré-natal (GRAVENA ET AL., 2013 SANTOS; MARTINS; SOUZA, 2008 RAMOS; CUMAN, 2009).

Foi no grupo de mulheres acima dos 35 anos onde encontramos o maior percentual de número de consultas adequada, que é de quatro a seis consultas durante a gestação, de acordo com o Ministério da Saúde (SANTOS ET AL., 2008).

Em relação a abortamento anterior $87 \%$ das adolescentes e $89 \%$ das mulheres acima dos 35 anos apresentaram esse fator de risco. No entanto, como foi observado na literatura, as mulheres geralmente sentem constrangimento ou medo em declarar abortos prévios, resultando em subnotificação de informações.

\section{CONCLUSÃO}

A partir dos resultados apresentados neste estudo, pode-se concluir que as variáveis relacionadas à idade, que implicaram no trabalho de parto prematuro, foram aborto prévio, primiparidade, sofrimento fetal, infecções do trato urinário e cobertura pré-natal inadequada na realidade pesquisada.

0 papel da enfermagem na prevenção do trabatho de parto prematuro e na minimização dos fatores riscos é de extrema importância. Os enfermeiros precisam ser preparados desde a graduação para uma assistência de qualidade ao pré-natal e à saúde da mulher em geral, além de uma visão crítica sobre a re- 
levância das orientações e educação em saúde como parte integrante do cuidar.

Além do aspecto inerente ao profissional de saúde, a rede de assistência à saúde deve garantir que a assistência pré-natal, condição que possibilita redução desses índices, seja oferecida de forma equânime a população. A realidade do local estudado nos mostrou, por meio dos dados encontrados, que essa assistência não está funcionando de acordo com os princípios do SUS.

O estudo possui algumas limitações como o registro insuficiente dos prontuários, o restrito tamanho da população e a realização da pesquisa em um único local. Com isso a generalização dos resultados em relação à população geral pode ser prejudicada.

No estado onde a pesquisa foi desenvolvida, e no município de Maceió não foram encontrados artigos publicados que aborde a mesma temática, então apesar das limitações metodológicas citadas acima, o valor do estudo é inegável, já que evidencia situações passíveis de prevenção como o número insuficiente de consultas pré-natal baseado no que é preconizado pelo ministério da saúde e o mau registro dos prontuários, conscientizando os profissionais de saúde sobre a importância da qualidade da assistência para a diminuição de fatores preveníveis do trabalho de parto prematuro principalmente nos extremos de idade reprodutiva.

\section{REFERÊNCIAS}

AGUIAR, A. S. C.; CARDOSO, M. V. L. M. L.; LÚCIO, I. M. $L$. Teste do reflexo vermelho: forma de prevenção à cegueira. Rev. Bras.Enferm., v.60, n.5, 2007. p.541-545.

ALMEIDA, A. C., JESUS A. C. P., LIMA, P. F.T., ARAÚJO, M. F. M., ARAÚJO, T. M. Fatores de risco maternos para prematuridade em uma maternidade pública de Imperatriz-MA. Rev Gaúcha Enferm., Porto Alegre
(RS), jun. 2012. 33(2):86-94. Disponível em: <seer. ufrgs.br/RevistaGauchadeEnfermagem/article/download/.../1949>. Acesso em:

ASSUNÇÃO, L. P. Fatores associados ao nascimento de pré-termo em Campina Grande, Paraíba, Brasil: um estudo caso-controle. Cad. Saúde pública, Rio de Janeiro, v.28, n.6, 2012. p.1078-1090.

BRASIL. Ministério da Saúde. Secretaria de Atenção a Saúde. Departamento de Ações programáticas Estratégicas. Gestação de alto risco: manual técnico. 5.ed. Brasília, DF, 2010.

CARNIEL, E. F., ZANOLLI, M. L., ALMEIDA, C. A. A., MORCILLO, A. M. Características das mães adolescentes e de seus recém-nascidos e fatores de risco para a gravidez na adolescência em Campinas, SP, Brasil. Rev. Bras. Saúde Matern. Infant., Recife, out./dez. 2006. 6 (4): 419-426. Disponível em: <http://www.scielo.br/ pdf/rbsmi/v6n4/09.pdf>. Acesso em: 14 ago. 2011.

DATASUS/SIAB. [site online]. Disponível em: <http:// tabnet.datasus.gov.br/cgi/tabcgi.exe?siab/cnv/SIABSbr.def>. Acesso em: 24 ago. 2011.

GRAVENA, A. F. et al. Idade materna e fatores associados a resultados perinatais. Acta Paul.Enferm., v.26, n.2,2013. p.130-135.

MADI, J. M. et al. Avaliação dos fatores associados à ocorrência de prematuridade em um hospital terciário de ensino. Rev. da AMRIGS, Porto Alegre, v.56, n.2, 2012. p.111-118.

NEME, B. Obstetrícia Básica. 3.ed. São Paulo: Sarvier, 2005.

NOMURA, M. L. et al. Colonização materna e neonatal por estreptococo do grupo B em situações de ruptura pré-termo de membranas e no trabalho de parto prematuro. Laborv. Bras. Ginecol. Obstet., v.31, n.8, 2009. p.397-403. 
PESQUISA Nacional de Demografia e Saúde da Criança e da Mulher 2006 [série online]. Disponível em: <http://bvsms.saude.gov.br/bvs/pnds/index.php>. Acesso em: 14 ago. 2011.

RAMOS, H. A. C.; CUMAN, R. K. N. Fatores de risco para prematuridade: pesquisa documental. Esc. Anna Nery Rev. Enferm., v.13, n.2, 2009. p.297-304.

SANTOS, G. H. N et al. Impacto da idade materna sobre os resultados perinatais e via de parto. Rev. Bras. Ginecol. Obstet., v.31, n.7, 2009. p.326-334.

SANTOS, G. H. N. S.; MARTINS, M. G.;SOUSA, M. S. Gravidez na adolescência e fatores associados com o baixo peso ao nascer. Rev. Bras. Ginecol. Obstet., v.30, n.5, 2008. p.224-231.
SEIDLER, S. L. L.Prevalência de intercorrências clínicas maternas relacionadas à prematuridade. Rev. contexto \& saúde, v.10, n.20, 2011. p.1251-1254.

SILVA, A. M. R. et al. Fatores de risco para nascimentos pré-termo em Londrina, Paraná, Brasil. Cad. Saúde Pública, Rio de Janeiro, v.25, n.10, 2009. p.21252138.

SILVA, L. A. et al. Fatores de risco associados ao parto pré-termo em hospital de referencia de Santa Catarina, Revista da AMRIGS, Porto Alegre, v.53, out-dez. 2009. p.354-360.

STOLL, B. J.; KLIEGMAN, R. M. 0 feto e o recém-nascido. Guanabara Koogan, Rio de Janeiro, 2002. p.447547.
1. Enfermeira, graduada pela Universidade Estadual de Ciências da Saúde - UNCISAL. E-mail: larissafeitosa125@hotmail.com

2. Enfermeira, graduada pela Universidade Estadual de Ciências da Saúde - UNCISAL. E-mail: castelamayra@hotmail.com

3. Enfermeira Obstetra, docente do Centro Universitário Tiradentes UNIT Maceió. E-mail: albambf@hotmail.com

4. Enfermeira Mestre em Ensino na Saúde, docente do Centro Universitário Tiradentes - UNIT Maceió. E-mail: apaularebelo@hotmail.com

5. Enfermeira Mestre em Enfermagem, docente da Universidade Estadual de Ciências da Saúde - UNCISAL. E-mail: fhernandapimpao@yahoo.com.br 
